This is the accepted version of the article:

Duval, D. ; González-Guerrero, A. B.; Dante, S. ; Osmond, J. ; Monge, R. ; Fernández, L.J.; Zinoviev, K. E.; Domínguez, C. ; Lechuga, L.M. . Nanophotonic lab-on-a-chip platforms including novel bimodal interferometers, microfluidics and grating couplers. Lab on a Chip - Miniaturisation for Chemistry and Biology, (2012). 12. 11: 1987 - . .

Available at: https://dx.doi.org/ 


\section{RSCPublishing Lab on a Chip}

\section{Nanophotonic lab-on-a-chip platforms including novel bimodal interferometers, microfluidics and grating couplers}

\begin{tabular}{|r|l|}
\hline Journal: & Lab on a Chip \\
\hline Manuscript ID: & Draft \\
\hline Drticle Type: & Paper \\
\hline Complete List of Authors: & n/a \\
\hline & $\begin{array}{l}\text { Duval, Daphné; CIN2, } \\
\text { González-Guerrero, Ana Belén; CIN2, } \\
\text { Dante, Stefania; CIN2, } \\
\text { Osmond, Johann; ICFO, } \\
\text { Monge, Rosa; GEMM-I3A, } \\
\text { Fernández, Luis; GEMM-I3A, } \\
\text { Zinoviev, Kirill; IMB-CNM, } \\
\text { Domínguez, Carlos; IMB-CNM, } \\
\text { Lechuga, Laura; CSIC, CIN2 }\end{array}$ \\
\hline \hline
\end{tabular}




\title{
Nanophotonic lab-on-a-chip platforms including novel bimodal interferometers, microfluidics and grating couplers
}

\author{
Daphné Duval, ${ }^{a}$ Ana Belén González-Guerrero, ${ }^{a}$ Stefania Dante, ${ }^{a}$ Johann Osmond, ${ }^{b}$ Rosa Monge, ${ }^{c}$ Luis J. \\ Fernández, ${ }^{c}$ Kirill E. Zinoviev, ${ }^{d}$ Carlos Domínguez ${ }^{d}$ and Laura M. Lechuga* ${ }^{a}$ \\ 5 Received (in $X X X, X X X)$ Xth $X X X X X X X X X 20 X X$, Accepted Xth $X X X X X X X X X 20 X X$ \\ DOI: $10.1039 / b 000000 x$
}

\begin{abstract}
One of the main limitations for achieving truly lab-on-a-chip (LOC) devices for point-of-care diagnosis is the incorporation of the "onchip" detection. Indeed, most of the state-of-the-art LOC devices usually require complex read-out instrumentation, losing the main advantages of portability and simplicity. In this context, we present our last advances towards the achievement of a portable and label10 free LOC platform with highly sensitive "on-chip" detection by using nanophotonic biosensors. Bimodal waveguide interferometers fabricated by standard silicon processes have been integrated with sub-micronic grating couplers for efficient light in-coupling, showing a sensitivity of $3.3 \cdot 10^{-7}$ refractive index unit (RIU) in bulk. A 3D network of SU-8 polymer microfluidics monolithically assembled at the wafer-level was included, ensuring a perfect sealing and a compact packaging. To overcome some of the drawbacks inherent to interferometric read-outs, a novel all-optical wavelength modulation system has been implemented, providing a linear response and a 15 direct read-out of the phase variation. Sensitivity, specificity and reproducibility of the wavelength modulated BiMW sensor has been demonstrated through the label-free immunodetection of the human hormone hTSH at picomolar level using a reliable biofunctionalization process.
\end{abstract}

\section{Introduction}

${ }_{20}$ One of the main challenges of the $21^{\text {st }}$ century is related to human health, including environment monitoring, food safety and early medical diagnostics. These fields share the common need of detecting very low concentration of analytes or toxins in realtime. Traditional techniques, such as ELISA or RIA tests, suffer 25 from important limitations: laborious sample preparation, the need of bulky instrumentation and the slow data processing. Moreover, they often have to be performed by specialized technicians in laboratory environments. To overcome these drawbacks, the implementation of a portable, easy-to-use and 30 highly sensitive biosensor lab-on-a-chip (LOC) for label-free and real-time analysis is mandatory. A LOC must combine, ideally, all the functionalities on a single chip: fluid handling, sample preparation (filtration, homogenization, dilution, etc.), target detection and signal processing. During the last years, the quest 35 for such fast and efficient analytical platforms has attracted the attention of many researchers. ${ }^{1,2}$

When dealing with a biosensor LOC system, the first issue to solve is the choice of the transducer. Even if the validity of electrochemical methods has already been demonstrated for 40 several applications, ${ }^{3,4}$ optical detection is generally considered as the most promising method for the development of label-free LOC platforms. In particular, integrated optical (IO) devices are having an increasing impact ${ }^{5-7}$ as they offer high sensitivity, mechanical stability, miniaturization and the possibility of mass45 production. They also present the advantages inherent to optical read-outs, i.e. non-invasive and non-destructive nature, absence of risk of electrical shocks or explosions and immunity to electromagnetic interferences. In addition, IO transducers have a great potential for parallel measurements making multiplexing 50 detection feasible.

Most of the IO sensors are based on the evanescent field detection principle: the biomolecular interaction between the analyte to detect and the corresponding bioreceptor immobilized on the waveguide (WG) surface within the evanescent field ${ }_{55}$ region results in a variation of the refractive index of the WG outer medium. This modification affects the effective index of the wave propagating in the WG which can be detected by measuring the intensity, the phase, the resonant momentum or the polarization of the output signal. In this detection scheme, there is 60 no need for labelling or prior separation of non-specific components making label-free detection feasible. Among the different IO sensors described in the literature or commercially available, it is worthwhile to mention the ones using micro-ring resonators, with a limit of detection (LOD) of 7,6 $10^{-7}$ refractive ${ }_{65}$ index unit (RIU) in bulk and $0,3 \mathrm{pg} / \mathrm{mm}^{2}$ in mass surface density, ${ }^{8}, 9$ grating couplers (LOD: $2,5 \cdot 10^{-6}$ RIU and 0,3 $\mathrm{pg} / \mathrm{mm}^{2}$ ), ${ }^{10}$ photonic crystals (LOD: $6 \cdot 10^{-4}$ RIU and 2,1 $\mathrm{pg} / \mathrm{mm}^{2}$ ), ${ }^{11}$ Young interferometers (LOD: $8,5 \cdot 10^{-8}$ RIU and 0,02 $\left.\mathrm{pg} / \mathrm{mm}^{2}\right)^{12}$ or Mach-Zehnder interferometers (LOD: $1 \cdot 10^{-7}$ RIU 70 and $\left.0,06 \mathrm{pg} / \mathrm{mm}^{2}\right){ }^{13}$

However, despite the growing interest of the academic and industrial communities, very few stand-alone LOC platforms based on IO sensors have emerged. Indeed, the transit from bulky and complex laboratory equipments to a small, portable and 75 cheap LOC device with low consumption is still a challenge: even if the technologies for each component are mature, their 
integration into a single platform is complex. The device must be carefully designed since it is not merely the sum of the basic units.

One key issue in the development of LOC devices is the way 5 to bring the sample in contact with the sensing area. The volume of the sample and the flow rate are critical parameters, especially for clinical testing where it is extremely important to reduce the sample volume. Recent technology efforts to combine the fields of photonics and microfluidics have led to the development of 10 optofluidics, in which optical and fluidic systems are integrated together to provide improved functions and performances. The interest in this field is demonstrated by the increasing number of papers and reviews published during the last years. ${ }^{14,15}$

In the case of IO sensors, the way to couple light into the WG 15 is another critical aspect as an efficient coupling increases the sensitivity of the device through an improved signal-to-noise ratio. Common in-coupling techniques are the end-fired method, the prism coupling and the grating assisted coupling, the last one being the most suitable for future commercial devices as it 20 provides better integration and stability. However, very few publications have demonstrated biosensing capabilities of IO sensors incorporating grating couplers. Exception is made with the multiplexed ring resonator array integrated with microfluidics and grating couplers developed by Carlborg et al. ${ }^{16}$ or the grating ${ }_{25}$ coupled with a WG interferometer recently optimized by Kozma et al. ${ }^{17}$

The biofunctionalization protocol is a third aspect which also plays a crucial role in the biosensor LOC performance as it will guarantee the sensitivity, the selectivity, the stability and the 30 longevity of the biosensor. Enormous efforts are continuously invested to develop new strategies adapted to a particular application and to each type of silicon-based photonic sensor. ${ }^{6,18}$

Taking into account all the above described aspects, different attempts have been done to integrate multiplexed IO biosensors 35 into LOC platforms and promising results have been achieved. ${ }^{19}$, ${ }^{20}$ But these systems still require complex read-out laboratory instrumentation, losing the advantages of portability and simplicity. Therefore, the main difficulty when implementing truly LOC devices for point-of-care platforms remains on the 40 incorporation of real "on-chip" detection.

To solve this gap, we present here our last advances towards the assembly of a LOC platform with "on-chip" detection using a recently developed nanophotonic biosensor, the bimodal waveguide (BiMW) interferometer. ${ }^{21}$ In the first part, the working ${ }_{45}$ principle and the fabrication processes of the nanometric BiMW will be presented as well as the experimental set-up. Then, the successful integration of several of the required units of a LOC platform will be demonstrated: (i) nanograting couplers for efficient light in-coupling into BiMW interferometers, (ii) a novel 50 all-optical wavelength modulation system to ensure linear response and avoid false read-out, (iii) reliable immobilisation protocols of the sensor surface and the immunosensing of the human hormone hTSH at pM level (iv) a 3D network of SU-8 polymer microfluidics monolithically assembled at the wafer ${ }_{55}$ level with the BiMW sensors.

\section{Bimodal waveguide interferometer}

Optical transduction principle

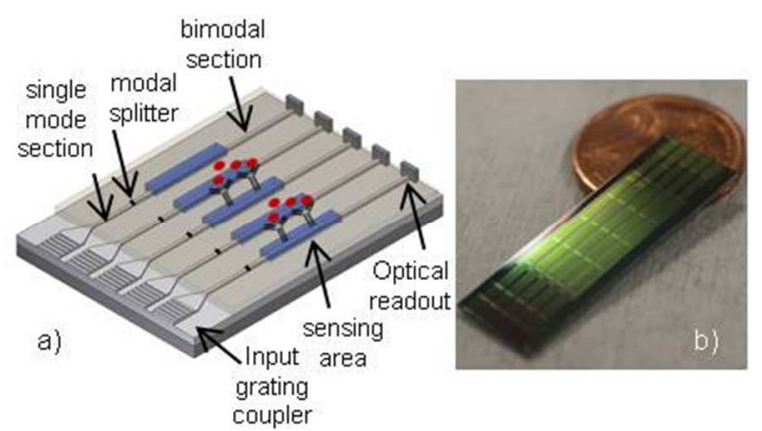

Fig. 1 (a) Scheme of the BiMW interferometers in a multiplexed 60 configuration, integrated with input grating couplers and photodetectors.

(b) Photograph of a chip of $30 \times 10 \mathrm{~mm}^{2}$ containing 16 BiMW interferometers.

In the BiMW sensor the interference of two WG modes (fundamental and first modes) of the same polarization occurs in ${ }_{65}$ a straight WG, without the need of a reference arm. ${ }^{21}$ The simplicity of this design using only straight WGs is especially attractive for the implementation of LOC platforms.

As depicted in Fig. 1a, the light from a coherent source is first coupled (by end-fire method or via a grating coupler) into a rib 70 WG that supports a single transversal mode. After a certain distance, the guided beam reaches a modal splitter that splits the first guided mode in two transversal modes, the fundamental and the first order modes, which are propagating until the output of the chip. A sensor area is defined on the bimodal part of the WG 75 and, as the fundamental and the first order modes have different intensity distributions at the core-cladding interface, the interference pattern is a function of the refractive index in the sensing region. In this scheme, there is no need for $\mathrm{Y}$ junctions facilitating the device fabrication as compared to other 80 interferometric configurations. Moreover, the interference pattern does not depend on the light intensity or on coupling variations which are important drawbacks when using Mach-Zehnder or Young interferometers.

Regarding multiplexing, the small foot-print of the BiMW 85 interferometer allows the integration of a large amount of sensing element within a single chip. For instance, Fig. 1b shows a chip of $30 \times 10 \mathrm{~mm}^{2}$ containing 16 BiMW distributed as four groups of four; up to 30 interferometers could be easily integrated in such chip.

\section{${ }_{90}$ Optical chip}

To optimize the sensor design, calculations and simulations were previously done as described elsewhere. ${ }^{21}$ According to the modelization, lithographic masks and devices were fabricated using standard microelectronics technology. The bottom cladding 95 layer consists of a $2 \mu \mathrm{m}$-thick thermally grown silicon dioxide layer $\left(\mathrm{n}_{\mathrm{SiO} 2}=1.46\right.$ at $\left.658 \mathrm{~nm}\right)$ deposited on a silicon wafer. Then, a $350 \mathrm{~nm}$-thick core layer of silicon nitride $\left(\mathrm{n}_{\mathrm{Si3N} 4}=2.00\right.$ at 658 $\mathrm{nm}$ ) is deposited by LPCVD. The thickness of the single mode section is reduced to $150 \mathrm{~nm}$ using conventional photolithography 100 and dry etching processes. Once the two different modal sections are defined, the nanometric rib structure of the WG $(4 \mu \mathrm{m}$ in width and less than $1.5 \mathrm{~nm}$ in height) is generated by BHF etching through a photoresist mask patterned by photolithography. The top cladding layer, a silicon dioxide layer 


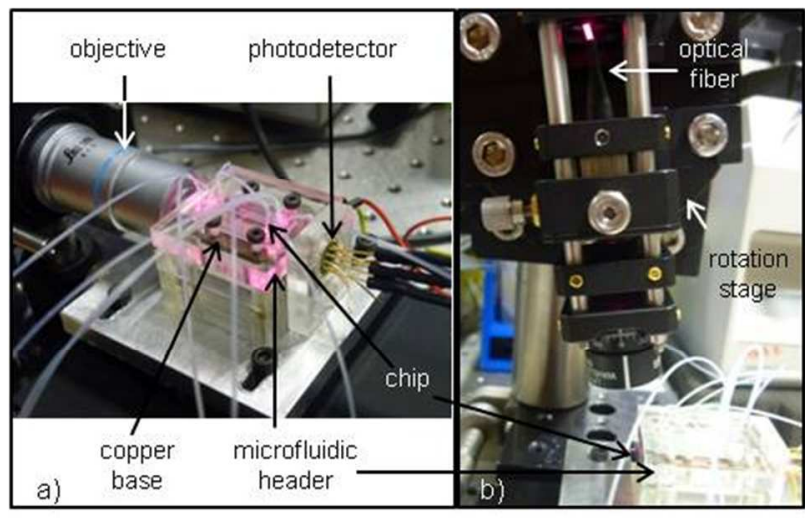

Fig. 2 Photographs of the set-up based on (a) end-fire method and (b) grating coupler method.

$\left(\mathrm{n}_{\mathrm{SiO} 2}=1.48\right.$ at $\left.658 \mathrm{~nm}\right)$, is then deposited by PECVD. The final 5 wet etching step defines the sensing window $\left(15 \times 0.05 \mathrm{~mm}^{2}\right)$ and the $2 \mathrm{~mm}$-long grating window.

The choice of a standard fabrication process offers important advantages such as robustness, reliability and potential for mass production with consequent reduction of costs. In addition, the 10 integration of light sources, photodetectors and CMOS processing electronics into the LOC platform would be facilitated if needed.

\section{Experimental set-up and procedures}

To control the temperature of the chip, a Peltier device, composed of a copper base and a thermo-electric element (TEC 3-2.5, 15 Thorlabs) connected to a temperature controller (TED 200C, Thorlabs), was integrated into a PMMA holder. The whole structure is placed on a XYZ translation stage (NanoMax, Thorlabs, travel range: $4 \mathrm{~mm}$, resolution $1 \mu \mathrm{m}$ ).

For light coupling into the chip, two different methods have 20 been used: end-fire coupling and grating assisted coupling. In the end-fire method, light from a high-power laser diode (ML101J27, Mitsubishi, $\lambda_{0}=658 \mathrm{~nm}, 120 \mathrm{~mW}$ ) is coupled into the WG using a $x 40$ microscope objective (Fig. 2a). For the grating coupler method, a fiber pigtailed laser diode (LPS-660-FC, Thorlabs, $\lambda_{0}=$ ${ }_{25} 658 \mathrm{~nm}, 7.5 \mathrm{~mW}$ ) is mounted on a rotation stage placed above the chip to control the incidence angle of the focused beam (Fig. 2b). In both cases, TE polarization is employed.

The interference pattern produced at the output of the BiMW is directly monitored by a two sectional photodiode (S5870, 30 Hamamatsu), each section being connected to a current amplifier (PDA 200C, Thorlabs). The currents generated in the upper and lower part of the photodetector, respectively $I_{u p}$ and $I_{\text {down }}$, are function of the interference pattern. To quantify the variation of this pattern, we define the sensor signal $S$ as

$$
S(\%)=\frac{I_{u p}-I_{\text {down }}}{I_{u p}+I_{\text {down }}} \times 100 .
$$

When a variation of the phase difference $\Delta \varphi_{S}$ is induced between the two guided modes, for instance by varying the refractive index in the sensing area, it results into an intensity variation of $S$ that can be expressed as:

$$
S(t) \propto \cos \left(\Delta \varphi_{S}(t)\right) .
$$

Table 1 Refractive indices of the $\mathrm{HCl}$ solutions used for calibration.

\begin{tabular}{ccc}
\hline Solutions & $\mathrm{n}$ & $\Delta \mathrm{n}$ \\
\hline $\mathrm{HCl} 0.03 \mathrm{M}$ & 1.3332 & $3 \cdot 10^{-4}$ \\
$\mathrm{HCl} \mathrm{0.05} \mathrm{M}$ & 1.3334 & $5 \cdot 10^{-4}$ \\
$\mathrm{HCl} \mathrm{0.1} \mathrm{M}$ & 1.3338 & $9 \cdot 10^{-4}$ \\
$\mathrm{HCl} \mathrm{0.2} \mathrm{M}$ & 1.3348 & $1.9 \cdot 10^{-3}$ \\
$\mathrm{HCl} \mathrm{0.3} \mathrm{M}$ & 1.3357 & $2.8 \cdot 10^{-3}$ \\
$\mathrm{HCl} \mathrm{0.4} \mathrm{M}$ & 1.3366 & $3.7 \cdot 10^{-3}$ \\
$\mathrm{HCl} \mathrm{0.5} \mathrm{M}$ & 1.3376 & $4.7 \cdot 10^{-3}$ \\
\hline
\end{tabular}

As it can be deduced from Eq. (2), the output of the BiMW device is periodic with respect to the phase changes induced in 45 the sensing area.

The microfluidic header used for preliminary measurements is a PDMS flow cell encapsulated in a PMMA housing to provide connections to the exterior. The microfluidic header has four independent channels of $3 \mu \mathrm{l}$ each, one on top of each of the four ${ }_{50}$ groups of interferometers.

Prior to biomeasurements, the sensitivity of the device is evaluated measuring the phase changes $\Delta \varphi$ induced by refractive index variations $\Delta n$ in the sensing area. Different concentrations of $\mathrm{HCl}$ (from 0.03 to $0.5 \mathrm{M}$ ) are injected with running water 55 (milli-Q grade) as buffer using a syringe pump. The refractive indices of the solutions have been previously measured with an ABBE refractometer (Optic Ivymen System). Table 1 reports the absolute refractive indices and the index difference induced in the sensing area considering milli-Q water $(\mathrm{n}=1.3329)$ as buffer.

60 The limit of detection (LOD) of the sensor is then estimated by considering that the lowest detectable phase $\Delta \varphi_{\min }$ is equal to three times the noise-to-signal ratio. In term of refractive index, the detection limit $\Delta n_{\min }$ is given by $\Delta n_{\min }=\Delta \varphi_{\min } / k$, with $k$ the slope of the calibration curve.

${ }_{65}$ We have previously reported a bulk refractive index sensing using BiMW interferometers of $2.5 \cdot 10^{-7} \mathrm{RIU}$, with a HeNe laser $\left(\lambda_{0}=633 \mathrm{~nm}\right)$ and end-fire coupling. ${ }^{21}$ Our previous result will be used as a reference to compare and estimate the performance of the BiMW device while it is progressively integrated with the 70 required elements of the LOC platform.

\section{Implementation of the lab-on-a-chip platform}

\section{Grating couplers}

How to couple light into a WG is an important issue to solve when dealing with LOC based on IO sensors. Our solution is to 75 integrate grating couplers at the input of the BiMW interferometers, making feasible better integration, stability and alignment tolerance than with the traditional end-fire method. Moreover, when coupling light with gratings, no polishing of the chip edges is required.

80 Due to the sub-micronic cross-section of the WG and to the operating wavelength $(\lambda=658 \mathrm{~nm})$, the grating length cannot exceed $100 \mu \mathrm{m}$ with a sub-micronic period which makes it suitable for high scale integration. To increase the coupling efficiency, the grating is associated to a tapered WG with an ${ }_{85}$ initial width of $20 \mu \mathrm{m}$ that linearly reduces until reaching the BiMW width $(4 \mu \mathrm{m})$. The resonant coupling between the guided TE mode and the grating is obtained when the following phase 


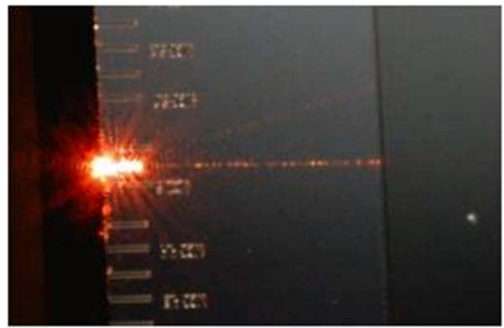

Fig. 3 Photography of the BiMW chip highlighting the excited grating and the light propagating in the WG.

matching condition is verified:

$$
{ }_{5} \sin \theta=n_{\text {eff }}+m \frac{\lambda}{\Lambda}
$$

with $\theta$ the incidence angle, $n_{\text {eff }}$ the effective index of the guided mode in the BiMW (here $n_{\text {eff }}=1.677499$ ), $m$ the diffraction order (here $m=-1$ ), $\lambda$ the wavelength and $\Lambda$ the grating period.

Eq. (3) is used to design gratings with incidence angles ranging 10 from $5^{\circ}$ to $15^{\circ}$. This range of angles enables an efficient coupling but, above all, it is an important requirement for the implementation of the complete LOC platform where the different units have to be integrated all together in a minimum of space but without disturbing each other.

15 Taking into account the above considerations, the gratings are directly written onto the $\mathrm{Si}_{3} \mathrm{~N}_{4}$ WG by electron beam lithography and reactive ion etching with a period of $450 \mathrm{~nm}$, a duty cycle of 0.5 and a depth of $50 \mathrm{~nm}$. The set-up shown in Fig. $2 \mathrm{~b}$ is used to excite the grating. An example of light coupled into a BiMW via 20 a grating is shown in Fig $3\left(\theta=9^{\circ} 50^{\prime}\right)$.

The sensitivity of the BiMW interferometer integrated with grating couplers is evaluated by carrying out a calibration curve with the set of solutions of $\mathrm{HCl}$ (Table 1). Fig. 4a shows the response of the system to an injection of a solution of $\mathrm{HCl} 0.2 \mathrm{M}$ 25 with milli-Q water as running buffer $\left(\Delta \mathrm{n}=1.9 \cdot 10^{-3}\right)$ : the phase starts at a constant level, corresponding to the running water, then oscillates when the $\mathrm{HCl}$ solution reaches the sensing area of the BiMW and finally stabilises. The total phase difference $\Delta \varphi$ equivalent to this change of index $\Delta n$ is estimated taking into 30 account that an entire oscillation is equivalent to a $\Delta \varphi$ of $2 \pi$. Here, the total phase difference for $\Delta n=1.9 \cdot 10^{-3}$ has been estimated to $3.89 \cdot 2 \pi \mathrm{rad}$. The same methodology has been applied to the other solutions and the resulting calibration curve is plotted in Fig. 4b. A linear fit gives a slope $k=2008 \cdot 2 \pi \mathrm{rad} / \mathrm{RIU}$, with ${ }_{35} \mathrm{R}^{2}=0.999$. With a noise-to-signal ratio of the system of $2.2 \cdot 10^{-}$ ${ }^{4} \cdot 2 \pi \mathrm{rad}$, a detection limit of $3.3 \cdot 10^{-7} \mathrm{RIU}$ is achieved. This LOD, obtained with a cheap and easily integrated laser diode, is comparable to the one demonstrated in our previous study using $\mathrm{HeNe}$ laser and end-fire method. ${ }^{21}$ This strongly supports the 40 adoption of the grating coupler method for the achievement of a truly portable and sensitive LOC platform.

It has to be noticed that in the case of BiMW interferometers, gratings for light out-coupling are not needed as we opted for a direct monitoring of the signal with a two sectional photodiode 45 directly placed at the WG output.

\section{All-optical phase modulation}
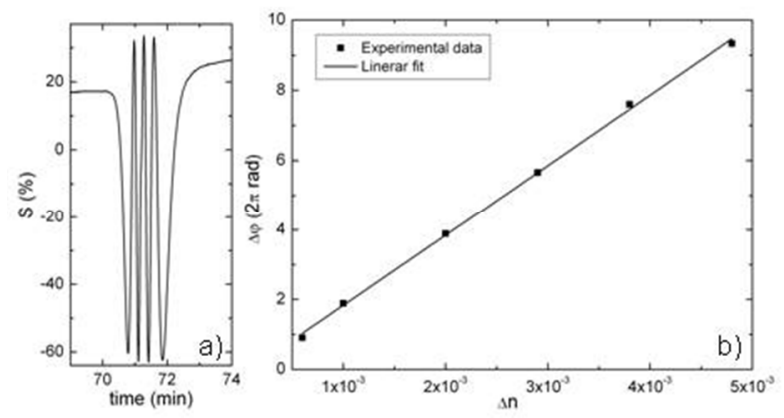

Fig. 4 (a) Sensor response in the case of an index change of $\Delta \mathrm{n}=1.9 \cdot 10^{-3}$ s0 and (b) calibration curve obtained with a BiMW excited via a grating coupler (TE polarization, $\theta=9^{\circ} 50^{\prime}$ ).

An important drawback of interferometric sensors comes from the periodic nature of the output signal which can give rise to wrong or ambiguous interpretations. This problem can be solved 55 by implementing a phase modulation system ${ }^{22}$ based for instance on electro-optical ${ }^{22}$, acousto-optical ${ }^{23}$ or magneto-optical ${ }^{24}$ working principles. However these techniques generally involve not standard CMOS materials and rely on complex read-out equipment which clearly impedes their integration into LOC ${ }_{60}$ platform. To overcome these difficulties, we recently have developed an all-optical modulation approach which provides a real-time and direct read-out of the phase variation without additional fabrication processes and instrumentation. ${ }^{25}$

In this modulation scheme, the phase difference between the ${ }_{65}$ two modes propagating in the BiMW is controlled by tuning few nanometers the input wavelength of the guided light. According to our calculations, a wavelength variation of $2 \mathrm{~nm}$ is enough to induce a shift of $2 \pi \mathrm{rad}$ between the two modes. This wavelength variation is easily obtained by taking advantage of a drawback of 70 the commercial Fabry-Perot laser diodes, i.e. the dependence of their emission wavelength with the driving current. Therefore, by applying a sinusoidal variation to the laser driving current, it is possible to induce a periodic phase change in the WG. As a consequence of this periodical change, the sensor output defined 75 by Eq. (2) is now expressed by:

$$
S(t) \propto \cos \left(\Delta \varphi_{S}(t)+\mu_{M} \sin (\omega t)\right)
$$

where $\mu_{M}$ is the amplitude and $\omega$ the frequency of modulation. A Fast Fourier Transform deconvolution of the output signal, performed in real-time with a Labview application, gives a direct 80 read-out of the phase variation. ${ }^{25}$

To implement this method, we used the end-fire coupling (Fig. 2a). The $2 \mathrm{~nm}$ wavelength shift was obtained with the laser diode ML101J27 (Mitsubishi). Fig. 5a shows the real-time sensor response during the injection of a solution of $\mathrm{HCl} 0.2 \mathrm{M}$ with 85 milli-Q water as running buffer $\left(\Delta \mathrm{n}=1.9 \cdot 10^{-3}\right)$ : the phase starts at a constant level, increases when the $\mathrm{HCl}$ solution reaches the sensing area and then stabilises. The difference between the two constant levels is the phase difference corresponding to the index variation in the sensing area (here, $\Delta \varphi=4.17 \cdot 2 \pi \mathrm{rad}$ ). With this 90 approach, the phase information is not deduced anymore from the variation of the interference pattern (as the one shown in Fig 4a) but is directly monitored, solving all the problems of false readout responses arising from interferometric signals. In addition, 

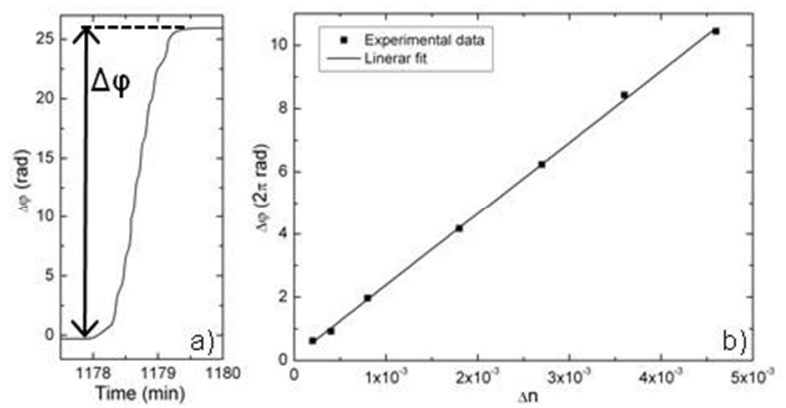

Fig. 5 (a) Real-time monitoring of the phase variation due to a change of $\Delta \mathrm{n}=1.9 \cdot 10^{-3}$ and (b) calibration curve of the wavelength modulated BiMW (TE polarization, laser driving current: $(143 \pm 51) \mathrm{mA}$, modulation 5 frequency: $215 \mathrm{~Hz}$ ) for different refractive indexes changes.

this detection scheme provides a theoretically infinite dynamic range of phase variation.

The phase changes for the complete set of $\mathrm{HCl}$ solutions have been evaluated in the same way. As a result, Fig. 5b shows the 10 calibration curve obtained with a wavelength modulated BiMW (TE polarization, laser driving current: (143 \pm 51) $\mathrm{mA}$, modulation frequency: $215 \mathrm{~Hz}$ ). A linear fit gives a slope $k=$ $2267 \cdot 2 \pi \mathrm{rad} / \mathrm{RIU}$, with $\mathrm{R}^{2}=0.999$. With the standard deviation of the system of $0.002 \mathrm{rad}$, a detection limit of $4.2 \cdot 10^{-7}$ RIU is 15 obtained. Again, this LOD achieved using cheap, compact and easily integrated laser diode is comparable to our previous results obtained with bulky HeNe laser. ${ }^{21}$ This, in addition to the direct phase read-out, clearly promotes the adoption of the all-optical wavelength modulation approach for the implementation of a ${ }_{20}$ LOC device.

\section{Biofunctionalization and biosensing evaluation of the wavelength modulated BiMW}

The biofunctionalization protocol plays a crucial role in the biosensor LOC performance as the immobilization process 25 should guarantee an efficient coverage of the transducer surface and ensure sensitivity, selectivity and stability of the biosensor. Different immobilization strategies can be employed according to the selected application, i.e. to the nature of the bioreceptor to be immobilized. However, one of the most reliable strategies is the 30 covalent binding through one of the chemical group of the molecules. ${ }^{6}$ In particular, for proteins, the preferred options are amino, carboxylic or thiol groups. To provide the sensor area with functional groups allowing covalent binding, silane molecules are extensively used.

35 The implementation of a suitable silanization method involves the optimization of different steps, such as the activation of the silicon surface by oxidation processes and the covalent anchorage of the silane molecule. In order to covalently attach proteins on the BiMW transducer, a carboxyethylsilanetriol sodium salt 40 silane (CTES, abcr) has been used. The formation of a stable and homogeneous monolayer of CTES supplies the silicon surface with carboxylic end-groups. The achievement of an enduring peptidic bond between the carboxylic groups present on the transducer surface and amino groups of the proteins is obtained 45 by the use of 1-ethyl-3-[3-dimethylaminopropyl] carbodiimide hydrochloride (EDC, Sigma-Aldrich) and Nhydroxysulfosuccinimide (NHS, Sigma-Aldrich).

We have selected as a clinical application the detection of the human Thyroid Stimulating Hormone (hTSH), secreted by the 50 anterior pituitary gland. The determination of low concentration of the hTSH is of clinical importance in the case of hyperthyroid syndromes such as thyroid adenoma, nodular goiter or the autoimmune disorder Graves' disease for which hTSH serum levels are below normal ones (normal concentration is considered 55 to be between $2-20 \mathrm{pM}$ ). To detect such low level, a competitive immunoassay was carried out with the wavelength modulated BiMW. In a competitive assay, the antigen to detect (here the hTSH) is immobilized on the sensor area surface. Afterwards, an antibody solution of an appropriate concentration is incubated 60 with the sample. The hormone concentration is then detected in an indirect way, by means of the signal obtained by the immunoreaction of the free antibodies in the solution with the immobilised hormones. This procedure implies more time and reagent consumption than direct methods but it allows the reuse ${ }_{65}$ of the bioreceptor surface many cycles thanks to regeneration solutions. In direct methods, the antibodies are immobilized on the sensor surface but after the biomolecular interaction, the use of regeneration solutions can easily denaturalize them. In a competitive immunoassay, several detections can be performed 70 using the same bioreceptor layer, reducing the total cost and duration when a complete analytical study is required.

In order to monitor in real time the interferometric signals obtained by the different steps of the biofunctionalization procedure, the processes are done in situ, with the BiMW chip 75 mounted with the microfluidic header on the experimental set up (Fig. 2a). During all the functionalization procedure, water flow is maintained at a constant rate of $10 \mu \mathrm{l} / \mathrm{min}$ and the different solutions are injected in a volume of $250 \mu \mathrm{l}$. The generation of reactive silanol groups on the sensor surface is achieved by 80 flowing a $20 \%$ nitric acid solution through the microfluidic channels. Three consecutive injections of the oxidizing solution were required to stabilize the interferometric signal. Then, a CTES solution at a concentration of $1 \%$ is employed to create a well-package monolayer of silanes. The ended carboxylic groups 85 of the silane are activated afterwards through the EDC-NHS chemistry. The hTSH is then immobilized by covalent bonding by supplying a $10 \mu \mathrm{g} / \mathrm{ml}$ solution of hTSH in phosphate buffer saline (PBS, $10 \mathrm{mM}$ phosphate, $2.9 \mathrm{mM} \mathrm{KCl}, 137 \mathrm{mM} \mathrm{NaCl}, \mathrm{pH}$ 7.4, Sigma-Aldrich). The hTSH solution is injected twice, to 90 assure the total covering of the surface by the proteins. Finally, a solution of ethanolamine (Sigma-Aldrich, $1 \mathrm{M}$, pH 8.5, SigmaAldrich) is injected in order to react with activated carboxylic groups, protecting the surface against unspecific adsorption. The buffer is then changed to PBS that is maintained during all the ${ }_{95}$ experiments at a constant rate of $20 \mu \mathrm{l} / \mathrm{min}$. Different solutions of the specific monoclonal antibody of hTSH, the anti-hTSH, are injected, with concentrations ranging from 0.5 to $5 \mu \mathrm{g} / \mathrm{ml}$. Regeneration of the biosensing surface is achieved by means of an injection of a $\mathrm{HCl}$ solution $(20 \mathrm{mM})$. The resulting calibration 100 curve, expressed as the phase variation as function of the antihTSH concentrations, is shown Fig. 6a.

Highly specific signals have been obtained as can be deduced form the control experiment presented in Fig 6b: the injection of a concentration of $5 \mu \mathrm{g} / \mathrm{ml}$ of anti-hTSH produces a phase 105 variation of $1.22 \cdot 2 \pi \mathrm{rad}$ while an injection of $5 \mu \mathrm{g} / \mathrm{ml}$ of the antibody against human growth hormone (anti-hGH), the control 

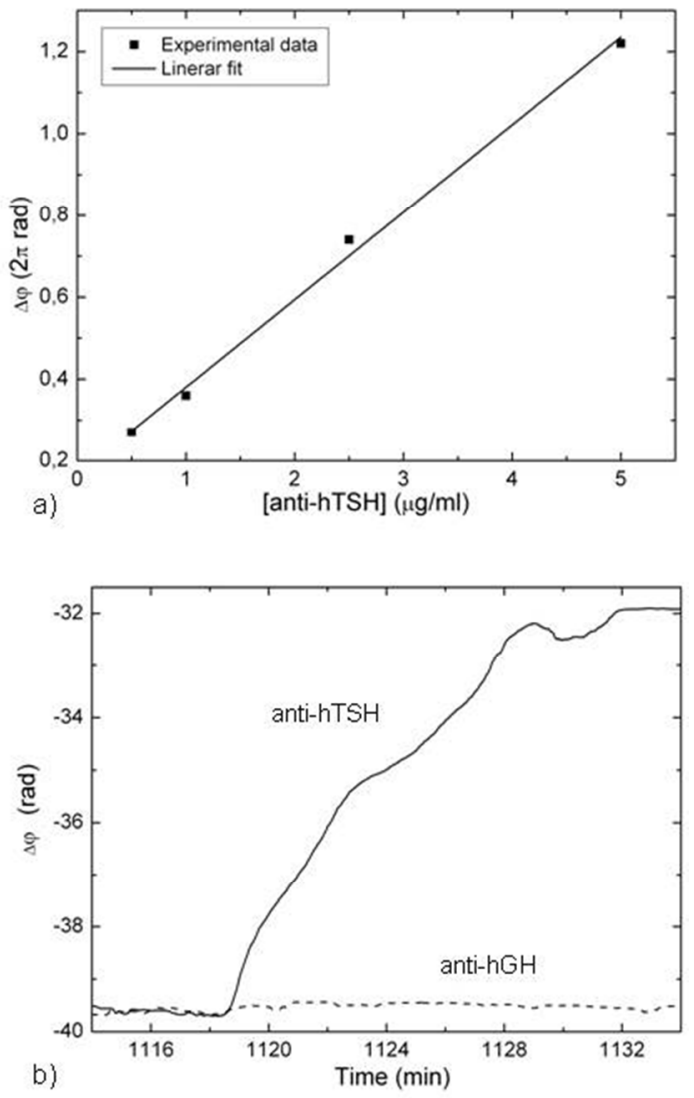

Fig. 6 (a) Calibration curve for the detection of different concentrations of anti-hTSH. (b) Control experiment: real-time monitoring of the phase variation for the detection of anti-hTSH $5 \mu \mathrm{g} / \mathrm{ml}$ and of anti-hGH $5 \mu \mathrm{g} / \mathrm{ml}$

5 employed as control. Results obtained with a wavelength modulated BiMW sensor (TE polarization, laser driving current: $(143 \pm 51) \mathrm{mA}$, modulation frequency: $215 \mathrm{~Hz}$ ).

antibody, does not induce significant phase variation, demonstrating the high selectivity of the immunoassay.

10 According to the above results, a fixed concentration of antihTSH of $1 \mu \mathrm{g} / \mathrm{ml}$ has been chosen to perform a competitive immunoassay. A $1 \mu \mathrm{g} / \mathrm{ml}$ solution of anti-hTSH is thus incubated with a $1 \mathrm{ng} / \mathrm{ml}$ hTSH solution and injected on the sensor surface. The results are shown in Fig. 7: the injection of $1 \mu \mathrm{g} / \mathrm{ml}$ of anti-

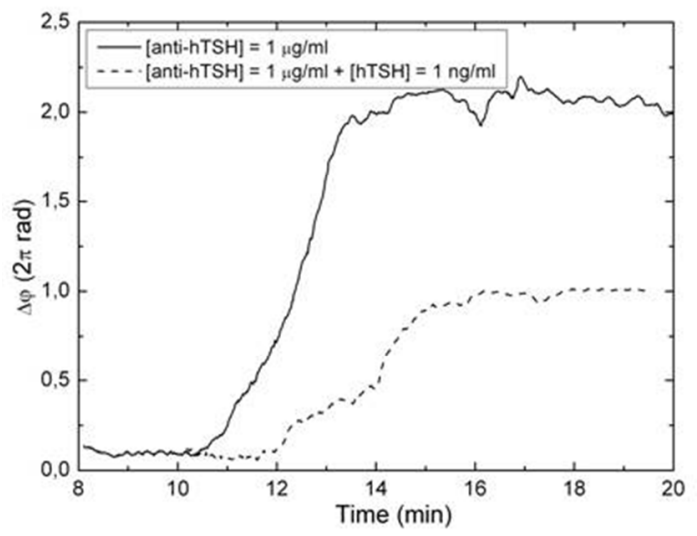

Fig. 7 Competitive assay: real-time monitoring of the phase variation for the detection of $1 \mu \mathrm{g} / \mathrm{ml}$ of anti-hTSH (solid line) and for the indirect detection of hTSH $(1 \mu \mathrm{g} / \mathrm{ml}$ of anti-hTSH $+1 \mathrm{ng} / \mathrm{ml}$ of hTSH) (dashed line).
20 hTSH induces a phase difference of $0.31 \cdot 2 \pi \mathrm{rad}$ while the injection of the incubated solution of $1 \mu \mathrm{g} / \mathrm{ml}$ of anti-hTSH with 1 $\mathrm{ng} / \mathrm{ml}$ of hTSH produces a phase difference of only $0.14 \cdot 2 \pi \mathrm{rad}$. This detection corresponds to a concentration of hTSH of $20 \mathrm{pM}$. However, given the significant phase difference between the two

25 signals, lower concentrations should easily be detected once the competitive assay protocols in situ will be further optimized.

Regarding reproducibility, the detection of anti-hTSH $1 \mu \mathrm{g} / \mathrm{ml}$ has been repeated three times. The sensor response was similar for the three evaluations: a standard deviation of $0.03 \cdot 2 \pi$ rad was 30 found for a mean value of $0.29 \cdot 2 \pi \mathrm{rad}$.

These results, obtained with a new wavelength modulated BiMW interferometer, are promising as they clearly demonstrate a high sensitivity, reproducibility and specificity.

\section{Microfluidics and Packaging}

35 In order to integrate a dedicated microchannel on each of the 16 sensors contained in each single chip, SU-8 based microfluidic technology was applied ${ }^{26-28}$. Figure 8 includes the schematic process flow used for the fabrication of the microfluidics devices. The process starts with the spinning of a $20 \mu \mathrm{m}$ thick SU-8 layer, 40 followed by a soft-bake step $\left(7\right.$ minutes at $90^{\circ} \mathrm{C}$ ). Then, another layer is spun and soft-baked in the same way to end up with a 50 $\mu \mathrm{m}$ thick layer (Fig. 8a). The increased thickness from the expected $40 \mu \mathrm{m}$ thick layer is caused by the difference in surface friction: while the first SU-8 layer is spun on top of silicon oxide, 45 the second SU-8 layer is spun on top of SU-8 material, increasing the final thickness. Once the final $50 \mu \mathrm{m}$ thick layer is obtained, an exposure of $140 \mathrm{~mJ}$ ultraviolet light (365 nm wavelength) is applied using a mask which defines $100 \mu \mathrm{m}$-wide microchannels. Then, a post-bake step is performed $\left(4\right.$ minutes at $\left.95^{\circ} \mathrm{C}\right)$ followed 50 by a gentle development during 4 minutes to remove the unexposed SU-8 (Fig. 8b). At this point, open microchannels are defined on top of every WG present on the chip. Since any debris deposited on top of the silicon nitride will dramatically decrease the resolution of the sensor, an oxygen plasma step was included 55 as an extra cleaning step (100 watt and $50 \mathrm{sccm}$ oxygen flow during 1 hour). The wafer was then measured by AFM to confirm that the silicon nitride sensing areas were completely SU-8 free.

To close the microchannels, another wafer with kapton on top was processed to carry a $40 \mu \mathrm{m}$ thick SU-8 layer (Fig. 8c). This 60 layer is meant to be the cover of the channels with the inlets and

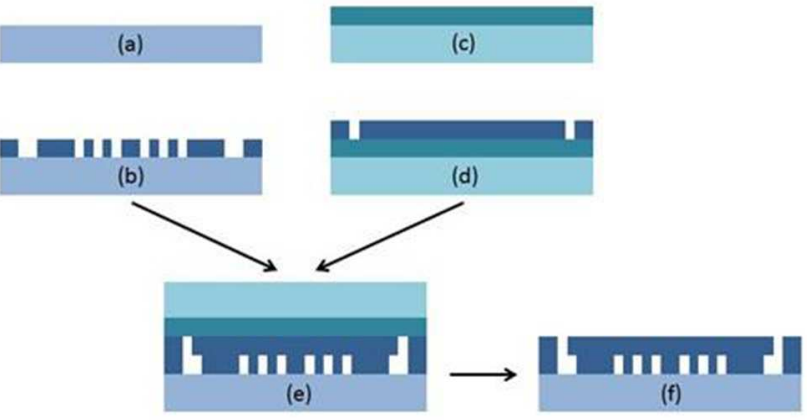

Fig. 8 Microchannel fabrication process flow: (a) spinning of SU-8 to reach a $50 \mu \mathrm{m}$ thick layer, (b) microchannel definition using photolithography, (c) kapton wafer with a $40 \mu \mathrm{m}$ thick SU-8 layer on top 65 (d) Cover definition by photolithography, (e) bonding step, (d) kapton wafer release 


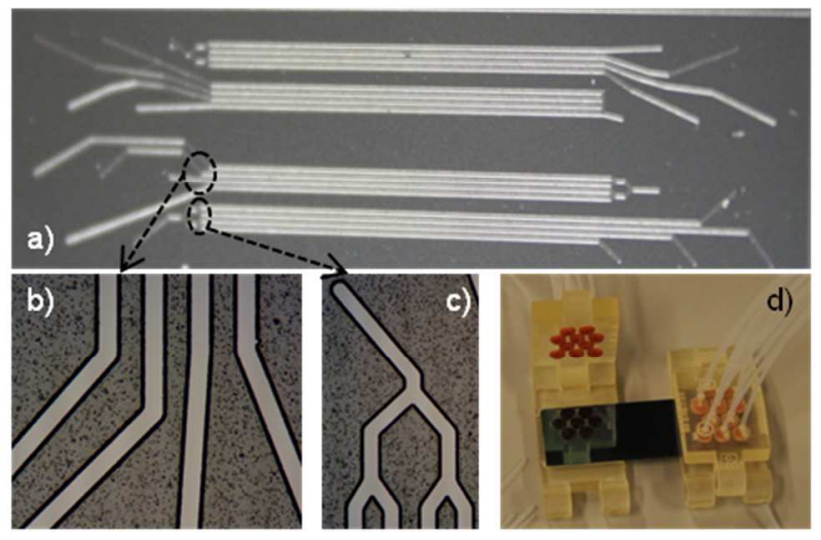

Fig. 9 Optical images of the 3D SU-8 microfluidic network: (a) BiMW chip with 16 channels, one on top of each sensor, (b) individual inlets of 4 sensors (c) common outlet of 4 sensors and (d) final encapsulation.

5 outlets already patterned by photolithography (Fig. 8d). After an alignment and a bonding step using a pressure of 3 bar at $90^{\circ} \mathrm{C}$ during 30 minutes (Fig. 8e), the kapton wafer was manually released from the silicon wafer thanks to its low adhesion to SU-8 (Fig. 8f). As a result, the microchannels were finally closed with 10 inlets and outlets accessible from the top of the wafer (Fig. 9a).

Due to the high density of microchannels present on the chip, the device and the packaging were designed to use one dedicated inlet for each microchannel (Fig. 9b), but a common outlet for every four sensors (Fig. 9c). In this way, only 20 fluidic 15 connections between the chip and the packaging were required to handle the device (instead of 32). As a result, the microfluidic connection between the outside and the sensor was done by the use of standard fluidic connectors and O-rings, ensuring a perfect sealing (Fig. 9d).

\section{${ }_{20}$ Conclusions and perspectives}

We are implementing a sensitive, affordable, hand-held and portable device for point-of-care diagnosis of relevant analytes. Our LOC platform is based on an innovative BiMW interferometer which has already shown its capabilities for ${ }_{25}$ sensitive, label-free and real-time biosensing. In contrast to most IO biosensors, the design of our interferometer is simple, allowing easy fabrication processes and integration.

Light in-coupling into the nano-interferometers using grating couplers has been achieved, leading to a limit of detection of $303.3 \cdot 10^{-7}$ RIU in bulk. A wavelength modulation system has been implemented in order to simplify data read-out and to solve some of the problems inherent to periodic signals. In addition, a versatile silanization method for surface biofunctionalization has been implemented and the detection of $20 \mathrm{pM}$ of hTSH has been 35 successfully demonstrated, showing high sensitivity, reproducibility and specificity. Regarding the microfluidic platform, a 3D microchannel network in SU-8 was fabricated at the wafer-level, ensuring a perfect sealing.

Our system presents a high degree of integration as sub40 micronic structures are employed in combination with compact laser diodes and simple read-out instrumentation. An additional advantage of our device lies in the use of standard silicon technologies together with polymer material processing, which could allow mass production with consequent reduction of costs.
45 Work is in progress to evaluate the surface sensing limit of detection of the wavelength modulated BiMW integrated with the grating couplers and the SU-8 microfluidic platform. Moreover, BiMW interferometers are currently being implemented in a multiplexed configuration, taking advantage of the individual ${ }_{50}$ inlet of the microchannel network to functionalize individually each BiMW sensor. The final prototype will incorporate the individual components presented here, plus commercial laser diodes, photodetectors, control electronics, firmware and software, sensor read-out algorithms and user interface.

\section{${ }_{55}$ Acknowledgements}

This work was partially developed using the micro and nano fabrication capabilities of the ICTS/clean room from the IMBCNM (CSIC) and has been sponsored by Fundación M. Botín.

The authors acknowledge A. García Castaño for the fabrication 60 of the PMMA microfluidic header.

S. Dante acknowledges the "Programa de Formación de Profesorado Universitario (FPU)" of the "Ministerio de Educación" of Spain.

\section{Notes and references}

${ }_{65}{ }^{a}$ Nanobiosensors and Bioanalytical Applications Group (CIN2) CSIC and CIBER-BBN, Campus UAB, Barcelona, Spain. E-mail:

laura.lechuga@cin2.es

${ }^{b}$ Institute of Photonic Sciences, ICFO, 08860 Castelldefels, Barcelona, Spain.

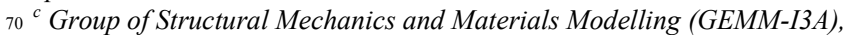

University of Zaragoza and CIBER-BBN, Zaragoza, Spain.

${ }^{d}$ Microelectronics National Center (IMB-CNM) CSIC, Campus UAB,

Barcelona, Spain.

75 1. C. D. Chin, V. Linder and S. K. Sia, Lab Chip, 2007, 7, 41-57.

2. F. S. Ligler, Anal. Chim. Acta., 2009, 81, 519-526.

3. M. A. Schwarz and P. C. Hauser, Lab Chip, 2001, 1, 1-6.

4. J. Wang, Biosens. Bioelectron., 2006, 21, 1887-1892.

5. P. V. Lambeck, Meas. Sci. Technol., 2006, 17, R93-R116.

80 6. M. C. Estevez, M. Alvarez and L. M. Lechuga, Laser Photonics Rev., 2011, DOI 10.1002/lpor.201100025.

7. A. Kussrow, C. S. Enders and D. J. Bornhop, Anal. Chem., 2011, 7, DOI 10.1021/ac202812h.

8. K. De Vos, J. Girones, T. Claes, Y. De Koninck, S. Popelka, E.

85 Schacht, R. Baets and P. Bienstman, IEEE Photonics J., 2009, 1, 225-235

9. D. X. Xu, M. Vachon, A. Densmore, R. Ma, A. Delâge, S. Janz, J. Lapointe, Y. Li, G. Lopinski, D. Zhang, Q. Y. Liu, P. Cheben and J. H. Schmid, Opt. Lett., 2010, 35, 2771-2773.

90 10. K. Cottier, M. Wiki, G. Voirin, H. Gao and R. E. Kunz, Sensor Actuat. B-Chem., 2003, 91, 241-251.

11. J. García-Rupérez, V. Toccafondo, M. J. Bañuls, J. G. Castelló, A. Griol, S. Peransi-Llopis and Á. Maquieira, Opt. Express, 2010, 18, 24276-24286.

95 12. A. Ymeti, J. Greve, P. V. Lambeck, R. Wijn, R. G. Heideman and J. S. Kanger, Appl. Optics, 2005, 44, 3409-3412.

13. K. E. Zinoviev, L. G. Carrascosa, J. Sánchez del Río, B. Sepúlveda, C. Domínguez and L. M. Lechuga, Adv. Opt. Tech., 2008, Article ID 383927, 1-6.

100 14. F. B. Myers and L. P. Lee, Lab Chip, 2008, 8, 2015-2031. 
15. X. Fan and I. M. White, Nat Photon, 2011, 5, 591-597.

16. C. F. Carlborg, K. B. Gylfason, A. Kazmierczak, F. Dortu, M. J. Banuls Polo, A. Maquieira Catala, G. M. Kresbach, H. Sohlstrom, T. Moh, L. Vivien, J. Popplewell, G. Ronan, C. A. Barrios, G. Stemme

5 and W. van der Wijngaart, Lab Chip, 2010, 10, 281-290.

17. P. Kozma, A. Hámori, S. Kurunczi, K. Cottier and R. Horvath, Sensors and Actuators B: Chemical, 2011, 155, 446-450.

18. J. T. Kirk, G. E. Fridley, J. W. Chamberlain, E. D. Christensen, M. Hochberg and D. M. Ratner, Lab Chip, 2011, 11, 1372-1377.

10 19. G. Suarez, Y.-H. Jin, J. Auerswald, S. Berchtold, H. F. Knapp, J.-M. Diserens, Y. Leterrier, J.-A. E. Manson and G. Voirin, Lab Chip, 2009, 9, 1625-1630.

20. A. Crespi, Y. Gu, B. Ngamsom, H. J. W. M. Hoekstra, C. Dongre, M. Pollnau, R. Ramponi, H. H. van den Vlekkert, P. Watts, G. Cerullo

15 and R. Osellame, Lab Chip, 2010, 10, 1167-1173.

21. K. E. Zinoviev, A. B. González-Guerrero, C. Domínguez and L. M. Lechuga, J. Lightwave Technol., 2011, 29, 1926-1930.

22. R. G. Heideman and P. V. Lambeck, Sensors and Actuators B: Chemical, 1999, 61, 100-127.

20 23. M. B. Duhring and O. Sigmund, Journal of Applied Physics, 2009, 105, 083529-083529-083529.

24. B. Sepúlveda, G. Armelles and L. M. Lechuga, Sensors and Actuators A: Physical, 2007, 134, 339-347.

25. S. Dante, D. Duval, B. Sepúlveda, A. B. González-Guerrero, J. R.

25 Sendra and L. M. Lechuga, Opt. Express, submitted.

26. F. J. Blanco, M. Agirregabiria, J. Berganzo, K. Mayora, J. Elizalde, A. Calle, C. Dominguez and L. M. Lechuga, J. Micromech. Microeng., 2006, 16, 1006-1016.

27. M. Castaño-Álvarez, M. T. Fernández-Abedul, A. Costa-García, M.

30 Agirregabiria, L. J. Fernández, J. M. Ruano-López and B. BarredoPresa, Talanta, 2009, 80, 24-30.

28. R. Vilares, C. Hunter, I. Ugarte, I. Aranburu, J. Berganzo, J. Elizalde and L. J. Fernandez, Sensors and Actuators B: Chemical, 2010, 147, 411-417.

35 
We show the implementation of a label-free LOC platform based on photonic interferometric sensors integrated with a novel alloptical wavelength modulation system, sub-micronic grating couplers and a SU-8 microfluidics network.

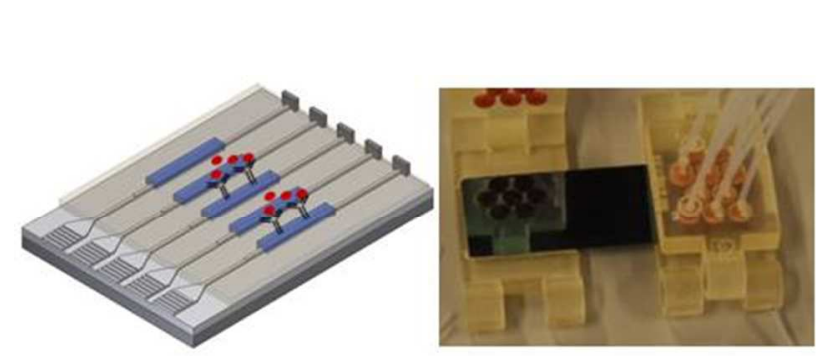 \\ Page 9 of 9}

